\title{
Computational Analysis of Climate Performance for Improving Design Strategies at an Australian Community
}

\author{
Yuqing Du \\ The University of Melbourne, VIC 3010, Australia \\ DOI: $10.32629 /$ aes.v2i4.545
}

\begin{abstract}
Nightingale 1 is a well-designed, community-led building for owner-occupiers in Brunswick, Melbourne, which is continuously awarded as the top five of the most livable cities in the World. To identify sustainability aspects of the Nightingale 1, the study will not only investigate local topographic characteristics and solar performance but also demonstrate the advantages or disadvantages of the current design related to the solar analysis. Based on the analysis, the study will propose improvements of thermal comfort in the housing project.
\end{abstract}

Keywords: thermal comfort, solar radiance, architecture design, climate responsive design

\section{Introduction}

Nightingale 1 is a well-designed, community-led building for owner-occupiers in Brunswick, Melbourne where is continuously awarded as the top five of the most livable cities in the world. Climatic comfort is one of the reasons why Melbourne is livable to many citizens who can enjoy an average temperature of $21^{\circ} \mathrm{C}$ in the hottest seasons and an approximate temperature of $10^{\circ} \mathrm{C}$ in the coldest seasons. The flat city develops with the rectangular pattern of streets decorated by a variety of public areas. The new proposals of property projects tend to launch in west and southeast regions far away from the Melbourne CBD while Brunswick is one of the northern suburbs closed to the Melbourne Central Area.

To identify sustainability aspects of the Nightingale 1, the study will not only investigate local topographic characteristics and solar radiance but also demonstrate the advantages or disadvantages of the current design related to the solar analysis. Based on the analysis, the study will propose improvements of thermal comfort in the housing project.

\section{Location}

Nightingale 1 is located in the north of Melbourne. Subtropical high prevails in the geographic position. The high pressure can suppress cloud formation that may turn into precipitation, giving rise to hot summers in the city (Song, 2017). However, an ocean is in the south of Melbourne and occasional cold anticyclones from the sea can raise warm air to form clouds for heavy precipitation. The anticyclone may also bring polar air mass, temperature thus plummets. Weather will be restabilized after the anticyclone dissipates. Another important topographic feature is that two blocks of plateaus sit in the north of Melbourne (see Figure 1). When the anticyclone spins and drives northerly wind in the Australian continent, thus crossing the canyon from the northern desert, extremely hot and dry air will rapidly heat the temperature in Melbourne.

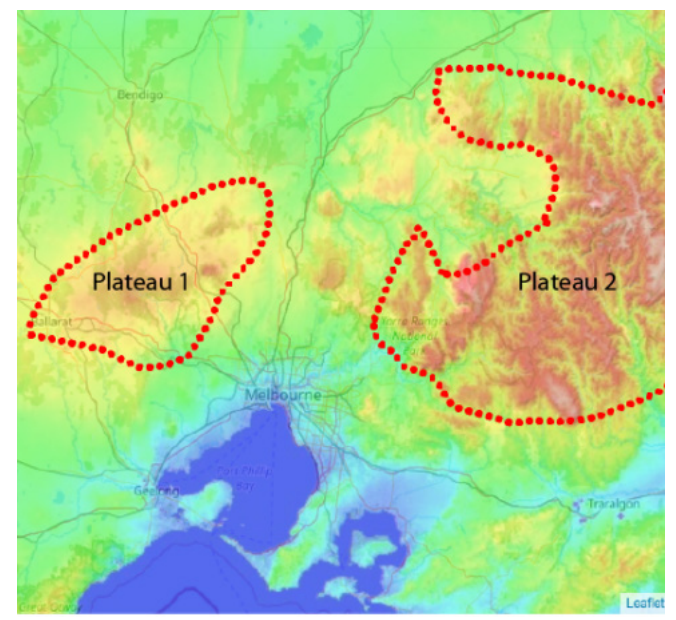

Figure 1. Two blocks of plateaus sit in the north of Melbourne

(Created by Yuqing Du. Referred to https://en-au.topographic-map.com/maps/jvbe/City-of-Melbourne/) 


\section{Solar analysis for climatic design}

\subsection{Point selections and the stereographic projection}

To specify the influence of various solar positions, the study selects two points in Nightingale 1 . They are separately located on the south and north face of the building (see Figure 2). Both of them are on the second floor due to the variation control. In comparison to Point B, Point A is in a southern smaller deck. It is potential to analyze the relationship among illuminated areas, lighting angles and solar altitude. The study makes use of the stereographic projection on the horizontal plane to explain the solar path. Based on De Troyer's view (2003), unlike deformation of projecting as an ellipse, such as compressed direction representations of other projections, leading to a misinterpretation, advantages of the stereographic projection, including an intuitive feeling of direction, the same projection of Zenith projection and of all points, can accurately interpret how solar irradiation can affect the Point A and Point B. Furthermore, it is feasible to illustrate the analysis chart by digital software or sketch because the sun paths are transformed in a circle on the sky vault rather than an ellipse. After analyzing the projection of sun paths and shadow angles in Point A (see Figure 3), the south-facing windows receive no direct sunlight in a whole year. The solar performance is a double-edged sword of climatic design. For example, residents living in this face might not suffer from exposure to the sun but cannot enjoy the sunshine. Comparatively, according to the projection chart of Point B (see Figure 4), sunlight cannot directly reach the north-facing windows in the winter solstice, June 21, from 11 am to $2 \mathrm{pm}$. The solar irradiation is less than 3 hours in May and July, and less than 2.5 hours in April and August. The insufficient irradiation takes place on low-temperature days, the sun, however, penetrates the north-facing windows on hot days. If the temperature rises persistently, the solar characteristic will threaten residents' thermal comfort in the "free-air-conditioner" architecture.

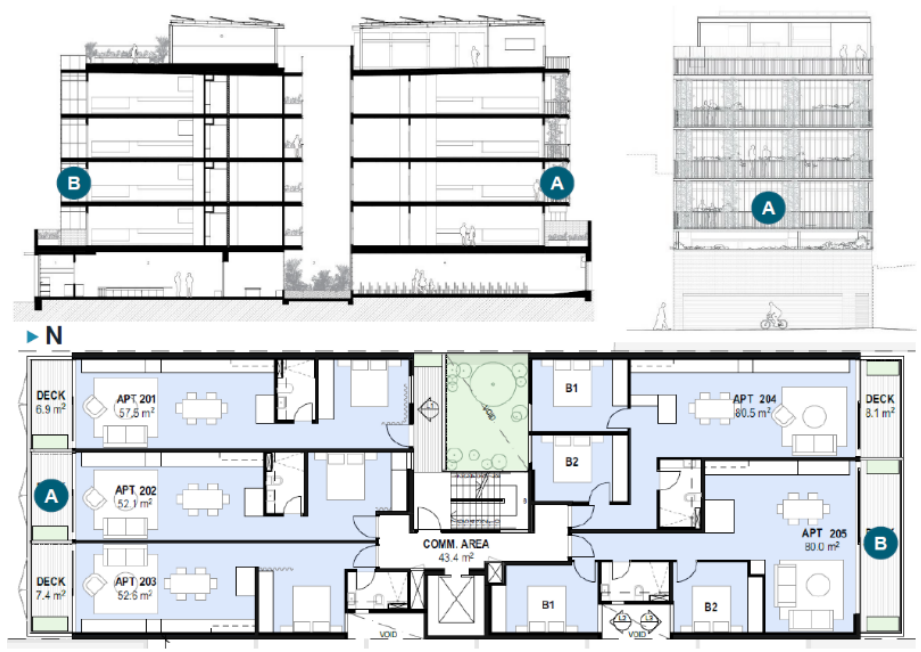

Figure 2. Location of selected Point A and Point B (Created by Yuqing Du. Referred to the engineering drawings by BREATHE Architecture)

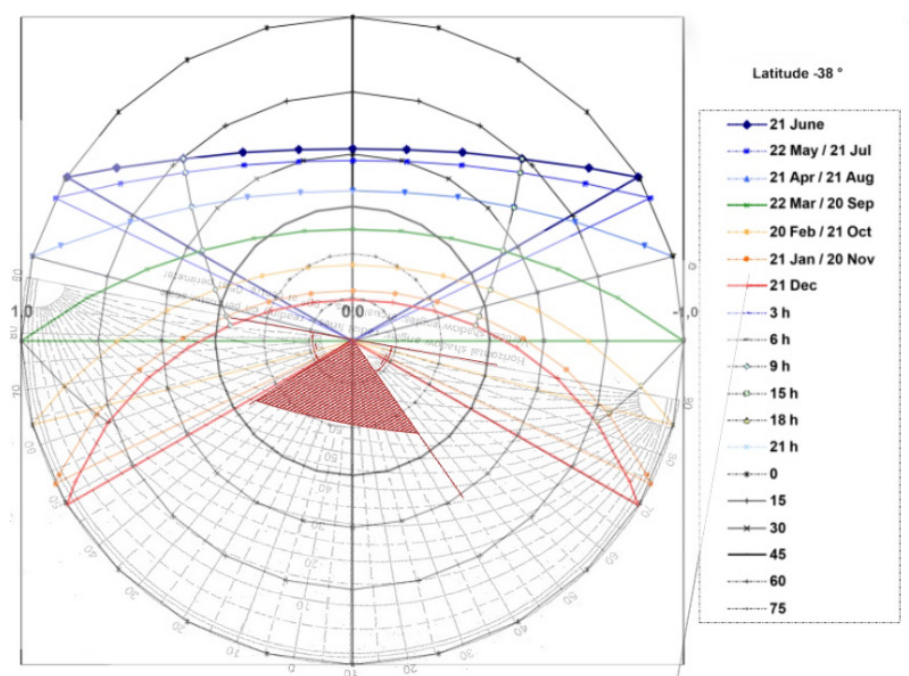

Figure 3. Stereographic projection of Point A (Created by Yuqing Du) 


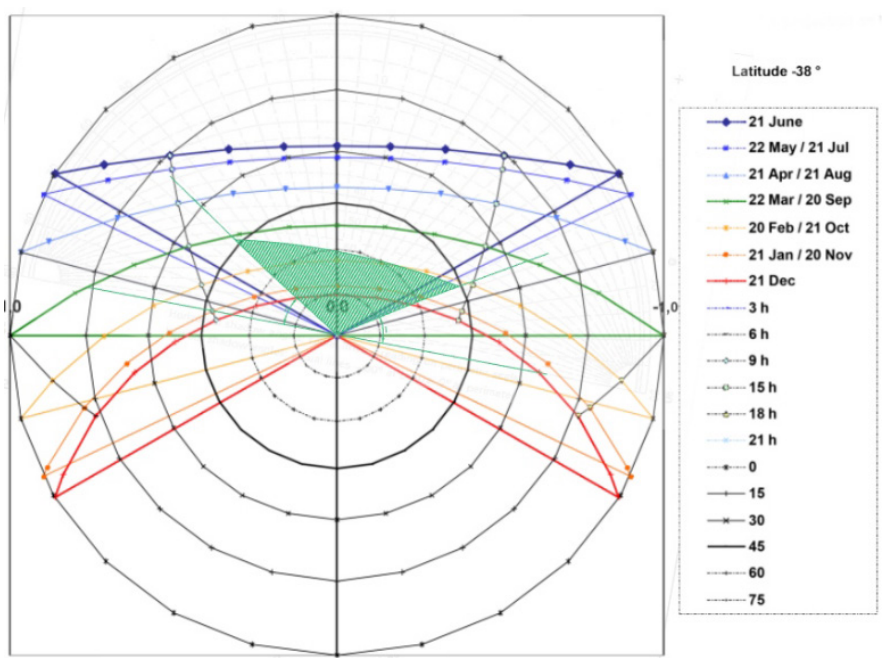

Figure 4. Stereographic projection of Point B (Created by Yuqing Du)

\section{Proposals of improvement}

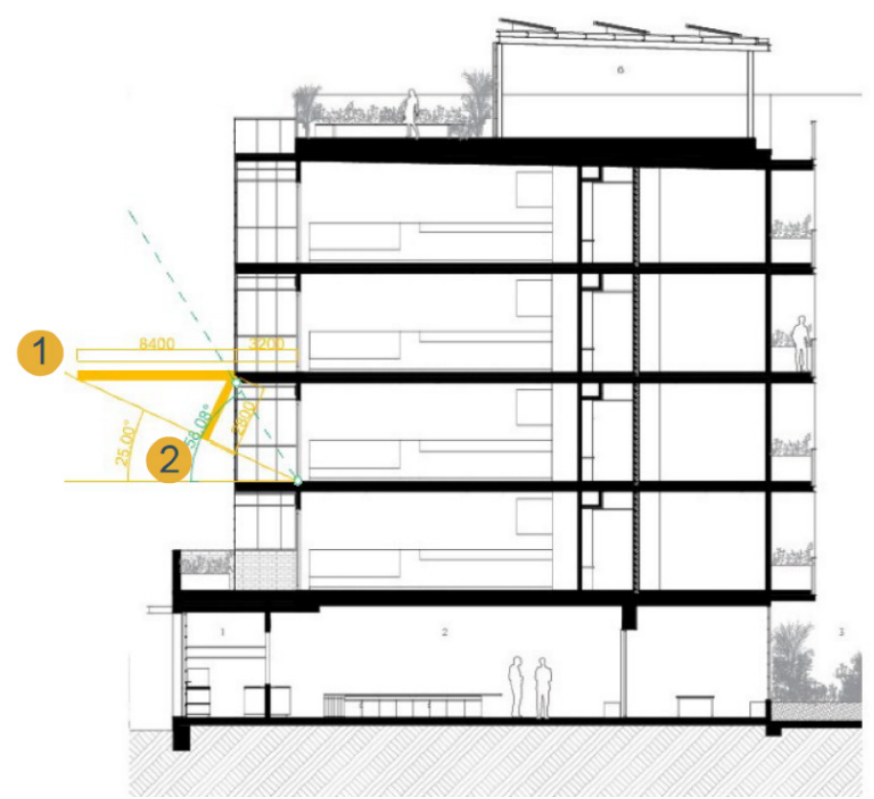

Figure 5. Proposed plans of changing angles of sun irradiation (Created by Yuqing Du. Referred to the engineering drawings by BREATHE Architecture)

The paper will offer several improvements from perspectives of thermal comfort and sustainable design. Considering the solar analysis and previous ventilation design, the shortage of sun irradiation in Point A cannot aggregate the temperature. The south-facing windows are open to cooling breeze from the southern ocean. However, in winter, for preventing heat loss in the room, it is more efficient to install double glazing windows or heavy curtains and pelmets. To improve the thermal experience on hot days for point $\mathrm{B}$, it is possible to avoid prolonged sun exposure by changing the irradiation angles into the deck. Although the existing linear pergolas embody lines of beauty and break direct sunlight in summer, they are not flexible to allow low-level irradiation in cold winter. The initial proposal of thermal comfort is extending shading devices and decks (see Figure 5). The scheme in Plan 1 is neither economical nor pragmatic to extend $8.4 \mathrm{~m}$. By comparison, rotating the extension in Plan 2 can achieve the same shading effect by installing an approximate $2.8 \mathrm{~m}$ roller shutter or awning bind. The installations are adjustable to provide external shading that should allow sunlight in winter. Awning blinds, roller shutters, or elastic linear pergolas are flexible to change forms related to the necessity of shading and basking. Additionally, deciduous trees can be a supplement to provide shading in summer but not block heating in winter. On the sustainable aspect, it is reasonable to find devices of solar energy in the south face of Nightingale 1 because of no direct sun irradiation. However, solar 
panels or devices of similar functions should have been visible in the north face of the building. The proposal of hidden solar panels behind windows will optimize the electricity supply in the Nightingale Housing model.

\section{References}

[1] De Troyer, F., (2003). Appropriate Sun-Path-Projection Tools: Correction of Intuitive Interpretation of 2d-Representations. In: Proceedings of the 20th Conference on Passive and Low Energy Architecture, Santiago - CHILE, Universidad Católica de Chile - PLEA, p 6.

[2] Song, C, (2017)., https://www.quora.com/How-does-the-weather-work-in-Melbourne-Australia-How-can-it-fluctuatefrom-so-cold-to-so-hot-and-back-so-quickly-and-why 